\title{
CLINICAL DEPARTMENT
}

\section{SARCOMA OF THE KIDNEY TREATED BY THE ROENTGEN RAY* \\ ALFRED FRIEDLANDER, M.D. CINCINNATI}

It is generally accepted as axiomatic that the only hope in cases of sarcoma of the kidney in childhood lies in early nephrectomy. Even under this procedure the mortality is very high on account of the likelihood of metastases, although the operation itself may be well borne.

The following case is reported because the growth was so large that no surgeon was willing to undertake its removal. For this reason recourse was had to treatment by the Roentgen ray. For a time there was marked improvement. The tumor became much smaller in size, and the child gained in weight and strength. After a time, however, the tumor began to increase in size again. Later, the child contracted measles, with complicating bronchopneumonia. At necropsy the actual results of the Roentgen ray treatment on the tumor mass could be studied. The report herewith submitted is presented because of the opportunity offered to study the effects of intensive Roentgen-ray treatment in a case of this sort.

W. S., boy, white, aged 4 years, was admitted to the pediatric service of the Cincinnati General Hospital on Oct. 20, 1915. The history was one of increasing languor and lassitude, with loss of appetite and anemia. The mother had noticed a mass in the abdomen three months before the child was brought to the hospital. A physician outside had suggested Roentgen-ray treatments. Two of these were given by a roentgenologist. After each of these treatments the boy vomited and passed almost pure blood for a day. This statement by the mother was later confirmed by the roentgenologist himself. For a time after the treatments the boy seemed better, but the improvement did not last.

On examination the child was found to be poorly nourished. Except for the condition of the abdomen, the physical findings were not of moment.

The entire left abdomen was filled by a tumor mass, extending from the costal margin in the nipple line to $3 \mathrm{~cm}$. above the symphysis. The tumor extended $1 \mathrm{~cm}$. to the left of the umbilicus. It was hard, distinctly nodular, apparently not tender to pressure and could be moved forward by pressure from behind.

Uranalysis on admission showed distinct microscopic hematuria. The blood showed a secondary anemia. Fluoroscopic examination with colon partly filled with gas showed a sharply defined dark shadow in the region normally occupied by the kidney. Roentgen-ray plate of the lungs for the characteristic metastatic sarcomatous shadows was negative.

* Submitted for publication May 21, 1916. 
In view of the fact that all of the surgeons who saw the child advised against operation because of the apparent hopelessness of the case, Roentgenray treatments were instituted.

Treatments were given with the Coolidge tube. Three areas, front, back and side of the tumor, were covered at each treatment, except the first and second, when one and two areas, respectively, were treated. Twenty treatments were given at intervals of about a week, with a dosage as indicated in the table. The distance in all treatments was 8 inches, with a spark gap of 9 inches.

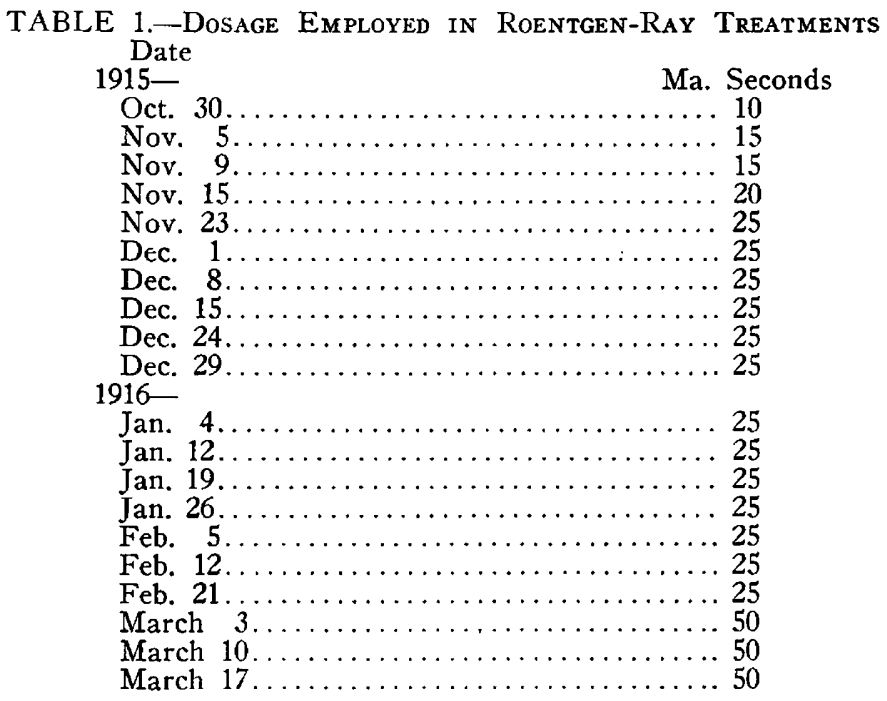

Before each Roentgen-ray treatment the child was given full doses of potassium citrate for a day. As a result of this alkalinization, no Roentgen-ray toxemia followed any of the treatments, except the last. Neither was there any increase of the blood in the urine after the treatments.

After the seventh treatment, given on December 8 , it was noted that the tumor had decreased very markedly in size. The child had gained several pounds in weight, looked rosy and well and played like an apparently normal child. In January the child had an attack of influenza, then epidemic in the wards, with double otitis media. The recovery was prompt. By March 1 the child was again very listless and apathetic. The tumor had grown appreciably in size. On March 30 the boy came down with measles, and death occurred on April 4.

The necropsy showed a sarcoma of the left kidney with small metastasis in both lungs and in the liver. The complete pathologic report is not given here.

At my request Prof. P. G. Woolley, pathologist to the hospital, made a microscopic examination of the tumor itself, with reference to the effects of the Roentgen ray on the growth. His report is herewith submitted:

Specimens for microscopic examination were removed from five different points in the tumor, one from near the upper pole, one from near the lower 
pole, one from the middle of the tumor at the edge of a hemorrhagic area, and the other two from intermediate portions. The capsule of the tumor was thick and hyaline like that seen in Zuckergussleber.

The stained sections showed the most widespread and generally diffuse necrotic changes, with no evidence of inflammatory reaction. Even the stroma showed degenerative changes, associated with irregular areas of edema. The parenchyma was almost completely necrotic, and, except in a few areas, chiefly near the lower pole, showed almost no evidence of structure. One could discern the remains of stroma and parenchyma, by means of the staining reactions, but all histologic cellular structures were lost. Karyorrhexis and karyolysis were obvious and the general appearances suggested those seen in areas of diffuse caseation. The capsule of the whole tumor mass was, on the other hand, hyaline, and, especially about the blood vessels, showed the structure of hyaline connective tissue. At no place was there any evidence of malignant cellular infiltration.

In the areas where some tumor structure persisted, the appearances were those of an alveolar sarcoma, and in these areas short spindle cells and round cells were present, chiefly the latter. In these areas the capillary vessels were healthy. About the margins of these tumorous foci the tumor cells and the interstitial cells were both changed. The former showed, first, pyknosis, and, as the areas of complete degeneration were approached, rhexis and swelling; the latter, edema and vacuolization.

The fact that the whole necrotic process was so widespread in such a large tumor mass; that there was no evidence of vascular thrombosis in the main vessels and no evidence of infarction; and that the degenerative process appeared to be a gradually progressive one, indicates that the Roentgen-ray treatments were at least partially the cause of the retrogression.

In view of some recently reported cases of hypernephromas of the kidney cured by the Roentgen ray it has seemed worth while to put on record this failure of the treatment in a sarcoma of the kidney in childhood.

Yet it should not be forgotten that the case was a particularly unfavorable one. Unquestionably, when a nephrectomy can be done with any prospect of success, it should be the procedure of choice. But it must be remembered that the mortality under surgical procedure is very high.

In view of the changes effected in the tumor mass by the Roentgen ray in this case (and the fact being admitted that one case does not permit the drawing of any sweeping conclusions), it seems justifiable to say that if nephrectomy is contraindicated in a case of sarcoma of the kidney, the Roentgen ray should be given a thorough trial.

4 West Seventh Street. 